\title{
Detection And Expression of a cDNA Clone That Encodes a Polypeptide Containing Two Inhibitory Domains of Human Calpastatin and its Recognition by Rheumatoid Arthritis Sera
}

\author{
Normand Després, Guylaine Talbot, Bertrand Plouffe, Gilles Boire, and Henri A. Ménard \\ Rheumatic Diseases Unit, Faculty of Medicine, Université de Sherbrooke, Sherbrooke, Québec, Canada, JIH 5N4
}

\begin{abstract}
RA is the most frequent and most destructive inflammatory arthropathy. Rheumatoid factors, in spite of their lack of disease specificity, are important serological markers for RA and appear important in its immunopathogenesis as well. In search of more disease-specific autoimmune systems, we have screened a human placenta $\lambda$ gt11 cDNA expression library using selected sera from patients with classical erosive RA. We have identified one clone (RA-1) that is recognized by three of five screening sera. The 950-bp insert shows a complete nucleotide sequence homology to the cDNA encoding the two COOH-terminal domains of calpastatin. The deduced open reading frame of the RA-1 cDNA predicts a 284-amino acid protein, with a calculated mol wt of $35.9 \mathrm{kD}$. Calpastatin is the natural inhibitor of calpains, which are members of the cysteine proteinases recently implicated in joint destruction in rheumatic diseases. The two domains encoded by the RA-1 clone each contain the structural features associated with the inhibitory activity of human calpastatin. By Western blotting, $45.5 \%$ or $21 / 44$ RA sera specifically recognized both the fusion and the cleaved recombinant protein. This is in contrast to $4.7 \%$ $(2 / 43)$ in nonrheumatoid sera and $0 / 10$ in normal sera. Anticalpastatin autoantibodies could represent a diseaseassociated marker in chronic erosive arthritis of the rheumatoid type and could hypothetically play a dual pathogenic role, directly via an immune interference and indirectly through an immune complex mechanism. (J. Clin. Invest. 1995. 95:1891-1896.) Key words: autoantibody • autoantigen - molecular cloning - cysteine proteinase inhibitor • autoimmunity
\end{abstract}

\section{Introduction}

Many manifestations of autoimmune diseases are characterized by their association with specific autoantibodies. In systemic

This work has been presented at the 1994 American College of Rheumatology National Scientific Meeting in Minneapolis, MN on October 2527, 1994 and has appeared in abstract form (1994. Arthritis Rheum. 37[ [Suppl.]:287a).

Address correspondence to Henri-André Ménard, Rheumatic Diseases Unit, Faculty of Medicine, Université de Sherbrooke, 3001 12th Avenue N., Sherbrooke, Québec, Canada, J1H 5N4. Phone: 819-5645261; FAX: 819-564-5265.

Received for publication 26 August 1994 and in revised form 5 December 1994.

J. Clin. Invest.

(C) The American Society for Clinical Investigation, Inc.

0021-9738/95/04/1891/06\$2.00

Volume 95, April 1995, 1891-1896 lupus erythematosus, anti-dsDNA and anti-Sm autoantibodies can both serve as disease markers while only anti-dsDNA antibodies seem to have a pathogenic implication (1). In Graves' disease and myasthenia gravis, antibodies respectively targeting the thyroid-stimulating hormone and the acetylcholine receptors may actually drive the disease process $(2,3)$. Conversely, antithyroglobulin, antimicrosomal thyroid peroxidase, and antistriated muscle autoantibodies appear to act only as disease markers.

Several autoantibodies have been reported to be more closely associated with RA (4-8). With the exception of rheumatoid factors, which are implicated in local immune complex formation and deposition, there is no evidence for a pathogenic role for these autoantibodies which may represent mere disease markers. A search for antigen-antibody systems more relevant to RA pathogenesis is thus warranted.

Isolation of cDNA clones encoding human proteins and peptides have been extensively used to identify new autoantigens (9). We undertook the immunoscreening of a human placenta $\lambda$ gt11 expression library with a pool of sera from five chronic erosive rheumatoid factor positive RA patients. In this study, we report that these sera recognized a cDNA clone (RA$1)^{1}$ encoding a protein corresponding to the two most $\mathrm{COOH}-$ terminal (residues 425-708) of the four inhibitory domains of calpastatin, the natural inhibitor of calpains (10). Calpains are calcium-dependent cysteine proteinases which are important intracellular activators in the signaling pathways and in triggering apoptosis $(11,12)$. They may also play a direct extracellular role in the destruction of connective tissue matrix and cartilage occurring in arthritic synovial joints (13). We also demonstrate that a significant proportion $(45.5 \%)$ of RA patients produce antibodies against the purified recombinant protein encoded by RA-1. These observations allow us to hypothesize that immune interference with the natural inhibition of these proteinases may have implications in the severe erosive features observed in a subgroup of RA patients.

\section{Methods}

Sera and antibodies. Sera were obtained from 44 patients who fulfilled the American College of Rheumatology criteria for RA (14) and were seen at the Rheumatic Diseases Unit of the Université de Sherbrooke, Sherbrooke, Québec, Canada. We used 53 control sera: 11 from systemic lupus erythematosus patients, 27 from osteoarthritic patients, 5 from patients with miscellaneous rheumatic diseases, and 10 from normal subjects. A pool of five RA sera positive for rheumatoid factor, antiperi-

1. Abbreviations used in this paper: GST, glutathione S-transferase GST-RA-1, fusion protein; IPTG, isopropyl- $\beta$-D-thiogalactopyranoside; RA-1, cloned cDNA insert; rRA-1, recombinant protein. 
nuclear factor, and anti-Sa autoantibodies while negative for other RAassociated autoantibodies (against SS-A(Ro), SS-B(La), U1RNP, A2 hnRNP, histones, and vimentin), were selected for cDNA library screening. Anti-Sa antibodies were detected by Western blotting using a semipurified human placenta extract, as described (8). These pooled sera were absorbed with Escherichia coli strain Y1090 lysates to deplete natural antibodies to bacteria, some of which are capable of crossreacting with autoantigens. A polyclonal goat antiserum specific for glutathione S-transferase (GST) (Pharmacia LKB Biotechnology, Inc., Uppsala, Sweden) was used to analyze the expressed fusion proteins.

Screening of cDNA library with patient sera. A human placenta $\lambda g t 11$ oligo(dT)-primed expression library of $2.4 \times 10^{6}$ clones (Clontech Laboratories Inc., Palo Alto, CA) was screened with the serum pool diluted $1 / 200$ in Tris-buffered saline containing $0.05 \%$ Tween 20 (15). This library was selected for its high number of recombinant clones and because placenta is a good source of Sa autoantigen, a specific disease marker for RA (8). Duplicate nitrocellulose filters (Hybound; Amersham Corp., Arlington Heights, IL) were used and only plaques positive on both sheets were further purified. Bound antibodies were detected with goat anti-human IgG $(\mathrm{H}+\mathrm{L})$ alkaline phosphatase-conjugated antibodies (Jackson ImmunoResearch Laboratories, Inc., West Grove, PA) and developed with 5-bromo-4-chloro-3-indoly/phosphate/nitroblue tetrazolium substrates (Promega Corp., Madison, WI). Positive clones were finally tested with the five individual RA sera from the pool of serum used for library screening and five sera from normal subjects.

DNA sequencing. Positive $\lambda g t 11$ phages were amplified by plate lysis (16) and DNA was isolated using a lambda purification kit (QIAGEN Inc., Chatsworth, CA). cDNA fragments were subcloned into the pUC19 vector at the EcoRI cloning site (New England Biolabs Inc., Beverly, MA). DNA sequencing was carried out with an A.L.F. ${ }^{\mathrm{TM}}$ automatic sequencer (Pharmacia LKB Biotechnology Inc.), according to the dideoxy technique of Sanger et al. (17), using reverse and forward fluorescent oligonucleotide primers (Pharmacia LKB Biotechnology Inc.). Sequences were aligned and analyzed using the GenBank/EMBL databases (NCBI/BLAST network service).

Expression and purification of the fusion and recombinant proteins. The cDNA fragments were subcloned from pUC19 into pGEX-4T-1 (Pharmacia LKB Biotechnology Inc.). The pGEX-4T-1 expression vector is constructed to give fusion polypeptides with an $\mathbf{N H}_{2}$-terminal GST carrier $(27 \mathrm{kD})$ and a thrombin cleavage site (18). The E. coli PR745 lon $^{-}$strain (New England Biolabs Inc.) was transformed with these constructs and the correct size and orientation of the cDNA insert was confirmed by restriction analysis with PstI digestion. Parental or recombinant pGEX-4T-1 transformants were grown at $37^{\circ} \mathrm{C}$ until $\mathrm{OD}_{595}$ reached $0.5-1.0$ and induced with $1 \mathrm{mM}$ isopropyl- $\beta$-D-thiogalactopyranoside (IPTG) for $2 \mathrm{~h}$. Cells were lysed and fusion proteins (referred to as GST-RA-1) were affinity-purified using glutathione Sepharose-4B (Pharmacia LKB Biotechnology Inc.), as described (18). Recombinant proteins (rRA-1) were obtained by removing the GST carrier using 10 cleavage units of thrombin per milligram of fusion protein (Sigma Chemical Co., St. Louis, MO).

SDS-PAGE and immunoblotting. Bacterial lysates, fusion, and recombinant proteins were subjected to electrophoresis on reducing SDSpolyacrylamide gels $(10 \%)$ and the protein bands were Coomassie blue stained or electroblotted onto nitrocellulose filters $(8)$. The blots were blocked with 5\% dry milk in PBS, $\mathrm{pH} 7.3$ and probed with an appropriate dilution of nonabsorbed serum in PBS-5\% milk. Bound antibodies were detected with Protein A-horseradish peroxidase (ICN Biomedicals, Inc., Costa Mesa, CA) diluted in PBS-0.5\% milk. Filters were developed using the enhanced chemiluminescence detection system (ECL; Amersham Corp.). Sera were coded and the immunoblots read blind. All sera were tested at least twice on each substrate; when the results were discordant for a given serum, the results of a third test was the one recorded. Sera giving indeterminate results (i.e., very faint bands) were read as negative.

\section{Results}

Isolation of the RA-1 cDNA clone. Approximately $2.5 \times 10^{6}$ plaques were immunoscreened and one strongly positive clone

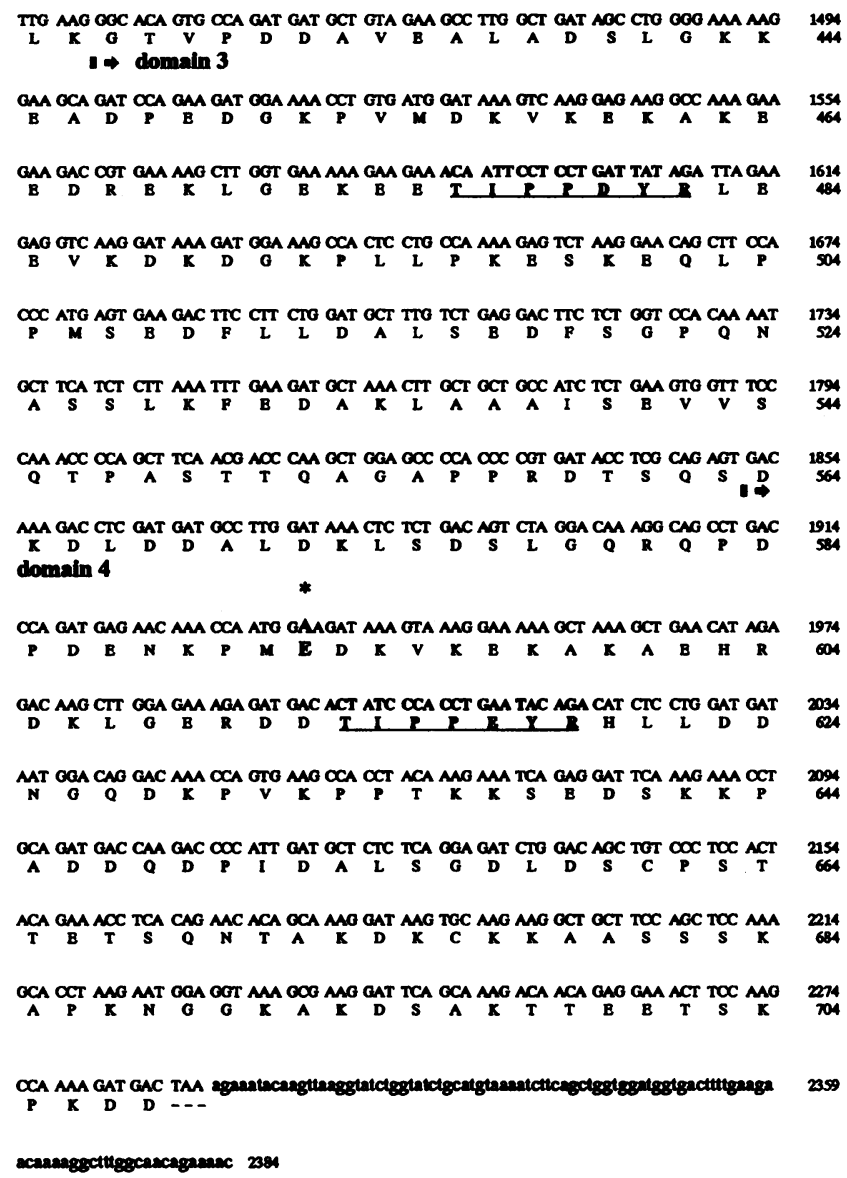

Figure 1. Nucleotide sequence of RA-1 cDNA and its deduced amino acid sequence. Numbers at the end of each line refer to nucleotide or amino acid positions as reported in the GenBank/EMBL Databank under accession number D16217. The deduced amino acid sequence of the encoded RA-1 polypeptide respected the reading frame of $\lambda \mathrm{gt} 11$ expression system and is displayed below the DNA sequence. A single nucleotide difference was observed between the $\mathrm{COOH}$-terminal calpastatin cDNA sequence and RA-1 cDNA sequence at position 1,937 resulting in a $G \rightarrow$ A nucleotide substitution at position 592 (indicated by an asterisk and bold capital letters). The boundaries of each domain are indicated by $(\|)$. Nucleotides and residues in boxes are the domains 3 and 4 functional consensus sequence (TIPPXYR), each associated with the calpastatin inhibitory activity. The termination codon $(T A A)$ is represented by $(--)$.

(RA-1) was identified and further characterized. The RA-1isolated cDNA insert was shown to contain $\sim 950$ bp by agarose gel electrophoresis. At this stage, we verified if the expressed $\beta$-galactosidase fusion protein from the purified $\lambda$ gt11 clone induced by IPTG reacted with individual sera. Three of the five rheumatoid sera recognized the positive plaque, whereas no reaction was detected with five nonrheumatoid control sera (data not shown). The cDNA insert was subsequently subcloned into pUC19 vector and sequenced. The 950-bp sequence of RA-1 was analyzed using the NCBI/BLAST network service and found to encode a 284-amino acid polypeptide (Fig. 1). The search for homologous sequences in the GenBank/EMBL databases showed an almost complete identity with the $\mathrm{COOH}$ terminal portion of human liver calpastatin at both the nucleo- 


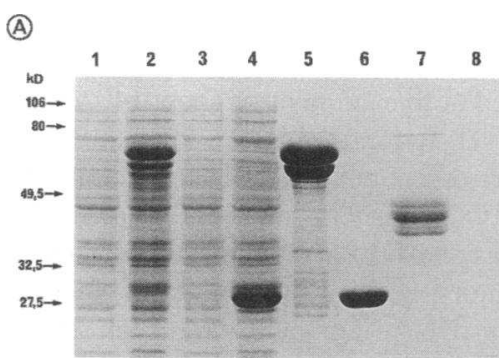

(B)

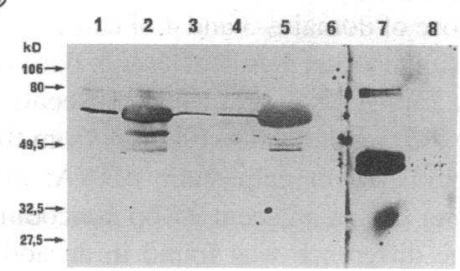

Figure 2. Purification of pGEX-4T-1-expressed recombinant proteins analyzed by SDS-PAGE $(A)$ and Western blotting (B). Recombinant proteins from $E$. coli PR745 lon $^{-}$containing pGEX4T-1/RA-1 or parental vector were induced, purified on glutathione Sepharose 4B, and digested with thrombin as described in Methods. Proteins were either Coomassie blue stained $(A)$ or transferred on nitrocellulose filters and antigenic proteins developed by Western blotting $(B)$. The Western blot was

performed using a pool of five nonabsorbed rheumatoid sera diluted 100-fold. Proteins were run separately in each lane: pGEX-4T-1/RA-1 transformant cell lysates before (lane 1 ) and after (lane 2) induction of GST-RA-1; parental pGEX-4T-1 transformant cell lysates before (lane 3 ) and after (lane 4) induction of GST alone; purified GST-RA1 (lane 5) and parental GST (lane 6) fractions eluted from glutathione Sepharose 4B; rRA-1 from the flow-through after thrombin cleavage of GST-RA-1 bound to glutathione Sepharose 4B (lane 7); and control thrombin cleavage of bound GST alone (lane 8 ).
(A)

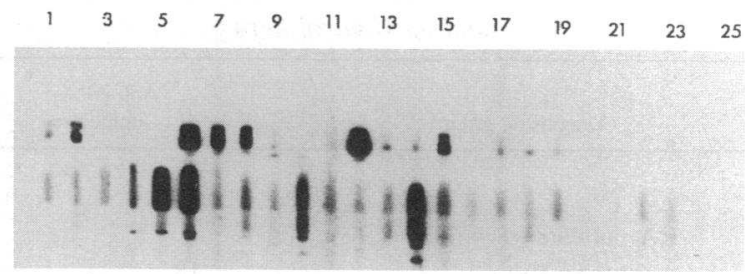

(B)

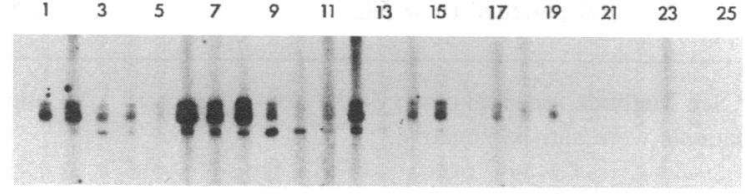

Figure 3. Recognition of GST-RA-1 $(A)$ and rRA-1 $(B)$ polypeptides using rheumatoid and nonrheumatoid sera. Affinity-purified GST-RA1 and cleaved rRA-1 polypeptide were separated on SDS-PAGE and transferred onto nitrocellulose sheets. These filters were probed with sera diluted at 1:100 for GST-RA-1 or at 1:50 for rRA-1. Lanes 1-19: serum from 20 patients with RA. Lanes 20-22: patients with systemic lupus erythematosus. Lanes 23-25: normal human sera. In $A$, sera detecting the two upper bands were considered positive. The strong signal below RA-1 bands likely represent $E$. coli contaminant proteins detected by natural antibodies. In $B$, the detection of the two upper RA-1 bands was considered a positive result. The results obtained with both fusion and recombinant RA-1 proteins were similar. Lanes 1, 2, 6-9, 12, 14 and 15 are strongly positive while lanes $3,4,11$ and $17-19$ are weakly positive.

strongly recognized by the serum pool (Fig. $2 \mathrm{~B}$, lane 5 ). A few lower mol wt bands were also immunoreactive and these may represent proteolytic degradation products. A $65-\mathrm{kD}$ band detected in both induced and uninduced parental pGEX-4T-1 (Fig. $2 B$, lanes 3 and 4 ) transformant cell lysates likely represented a bacterial protein recognized by patient sera. This protein was not induced by IPTG (Fig. $2 A$ and $B$, lane 4 ) and it was not affinity purified from glutathione Sepharose-4B (Fig. $2 A$ and $B$, lane 6 ). Thrombin cleavage of rRA-1 from its GST carrier resulted in a few $40-47-\mathrm{kD}$ bands (Figure $2 A$, lane 7), two of which were strongly recognized by the pool of RA sera (Fig. $2 B$, lane 7) and by its individual component sera (e.g., Fig. $3 B$, lanes 1 and 2 ). Although, the predicted mol wt of rRA-1 is $36 \mathrm{kD}$, the two major antigenic bands migrated as proteins of 43 and $47 \mathrm{kD}$ (Fig. $2 \mathrm{~B}$, lane 7).

The 75-kD polypeptidic band (Fig. $2 B$, lane 7) is an $E$. coli heat-shock protein produced by the dnaK gene that copurifies with the recombinant protein (20) and is recognized by natural antibodies in some nonabsorbed patient sera. The reactivity against this protein could be abolished after $E$. coli absorptions of the sera without depletion of anti-rRA-1 antibodies (data not shown). A protein with an apparent $M_{\mathrm{r}}$ of $40 \mathrm{kD}$ was recognized by some sera (Fig. $3 B$ ), independently of the recognition of the two rRA-1 bands. For example, in Fig. $3 B$, the serum in lane 1 only recognized the $43-$ and $47-\mathrm{kD}$ rRA-1 doublet, the serum in lane 10 recognized only the lower $40-\mathrm{kD}$ band, while the sera in lanes 6-9 recognized all three bands. This suggests that the $40-\mathrm{kD}$ band is not antigenically related to rRA-1. The absence of recognition of GST-RA-1 by sera recognizing only the $40-\mathrm{kD}$ band in the $\mathrm{rRA}-1$ preparation further supports this interpretation (Fig. $3 A$ and $B$, lane 10). 
Table I. Detection by Immunoblotting of Anti-RA-1 and Anti-Sa Antibodies in $44 R A$ and 53 Control Sera

\begin{tabular}{ccc}
\hline Diagnostic group* & \multicolumn{2}{c}{$\begin{array}{c}\text { Anti-rRA-1 } \\
\text { antibodies }\end{array}$} \\
\hline & + & - \\
RA patients $(n=44)$ & & \\
Anti-Sa $(+)$ & 18 & 10 \\
Anti-Sa $(-)$ & 3 & 13 \\
Non-RA patients ${ }^{\ddagger}(n=53)$ & 2 & 51 \\
\hline
\end{tabular}

* See Methods for breakdown of the non-RA group. ${ }^{\ddagger}$ All non-RA patients were anti-Sa negative.

Finally, the pool of sera failed to react with GST (Fig. $2 B$, lanes 4 and 6 ). Taken together, these results showed a strong reactivity of RA sera against the RA-1 polypeptides with a lack of detection of the GST moiety.

Reactivity of rheumatoid and control patients with fusion and recombinant $R A-1$. To determine whether individual sera from RA patients reacted with the RA-1 polypeptides, Western blots were performed using purified GST-RA-1 and rRA-1 (Fig. 3 ). The results with both proteins were similar. The two rRA1 polypeptidic bands $(43 \mathrm{kD}$ and $47 \mathrm{kD})$ were recognized by 21 of 44 RA sera $(45.5 \%)$ vs two of $43(4.7 \%)$ nonrheumatoid sera and none of 10 normal sera. The two nonrheumatoid sera had the clinical diagnosis of osteoarthritis. These data show that patients with rheumatic diseases produce autoantibodies recognizing a polypeptide (rRA-1) that corresponds to two functional domains of calpastatin. They also suggest that the production of anti-rRA-1 antibodies may be preferentially, but not exclusively, associated with RA.

Because the library screening sera were anti-Sa positive and because the prevalence of anti-RA-1 and anti-Sa antibodies (8) in RA were similar, the 97 sera were also tested for anti-Sa antibodies by Western blot (Table I). 31 RA sera were concordant ( 18 both positive and 13 both negative) and 13 were discordant ( 3 anti-RA-1 positive only and 10 anti-Sa positive only). The 53 control sera were all anti-Sa negative. Because these studies did not use full length calpastatin or pure $\mathrm{Sa}$ as antigens, it would be premature to conclude on the presence or absence of a molecular relationship between the two autoimmune systems.

\section{Discussion}

Autoantibodies to intracellular components expressed on cell surfaces or secreted in the extracellular environment are potentially pathogenic $(1-3,21)$. There is substantial evidence that many disease-associated autoantibodies have the ability to interact directly with enzymes and downregulate their activity (1, 21 ). Recent reports further suggest that autoantibodies can also upregulate enzymatic activity through interaction with proteinase inhibitors. Thus, anti-C1-inhibitor antibodies have been detected in patients with severe episodes of angioneurotic edema and acquired $\mathrm{C} 1$ deficiency (22). A subpopulation of antiproteinase 3 antibodies from patients with Wegener's granulomatosis can block the interaction of this serine proteinase with its natural inhibitor alpha 1-antitrypsin $(23,24)$. The present report provides another example of autoantibodies targeting a proteinase inhibitor.

Calpastatin is composed of five domains of about 140 amino acid residues each. The $\mathrm{NH}_{2}$-terminal domain (domain $\mathrm{L}$ ) is nonhomologous to the four others and per se has no inhibitory activity. Each of the four $\mathrm{COOH}$-terminal domains (domains 1 4 ) is repetitive and contains one TIPPXYR consensus sequence associated with the inhibitory activity of calpastatin. Indeed, using subcloned calpastatin cDNA fragments, it has been shown that each of the four homologous domains can block the proteinase activity of calpains (25). The RA-1 clone encodes two of the inhibitory sequences, those of domains 3 and 4 of calpastatin (Fig. 1). Strong evidence suggest that RA-1 cDNA is derived from the calpastatin mRNA. First, only 1 out of 950 consecutive bp (i.e., a 99.89\% identity) differentiates RA-1 cDNA from the published $3^{\prime}$ end of the human liver calpastatin CDNA; this identity spans both the coding and an adjacent 95-bp noncoding sequence. Second, the same difference was found in an additional cDNA encoding domain 4 of calpastatin and all the $3^{\prime}$ noncoding region, including the polyadenylation signal and the poly-A tail, that we recently cloned using a RA-1 cDNA-derived probe (data not shown). Third, this single nucleotide difference is not found when pig and rabbit calpastatin cDNAs are looked at. These data suggest either a polymorphism of the calpastatin mRNA or the existence of a sequencing mistake in the GenBank entry for human calpastatin. Thus, the immunoreactivity against RA-1 raises the hypothesis of specific immune interference with the interaction between calpastatin and calpains. However logi$\mathrm{cal}$, this proposition remains to be demonstrated.

Proteolytic enzymes are thought to play an important role in joint destruction. These enzymes belong to three major families: the matrix metalloproteinases, the serine proteinases, and the cysteine proteinases (26). The relative importance of these proteinases and their respective inhibitors in matrix destruction remains to be definitely established in vivo. However, recent data suggest that cysteine proteinases, particularly the calpains and their natural inhibitor calpastatin, could be important (2729). First, calpains are present in increased quantity in the synovial fluid (30-34) and membrane (13). Second, they are secreted in vitro by TNF $\alpha$ and IL-1-stimulated synovial fibroblast-like cells (35). Third, calpains are capable of degrading matrix components of articular cartilage and calpastatin can inhibit this degradation $(29,31,32)$. Fourth, a role for synthetic cysteine proteinase inhibitors as disease-modifying antirheumatic agents has been recently proposed in adjuvant-induced arthritis of rats and in collagen-induced arthritis of mice (36).

Under normal and pathological conditions, proteinases from each major family are inactivated by their natural inhibitors: tissue inhibitors of metalloproteinases for matrix metalloproteinases (37), serpins for serine proteinases (38), and the cysteine proteinase inhibitors. The natural cysteine proteinase inhibitors are members of a superfamily including the cystatin, the stefin, and the kininogen families. Calpastatin does not belong to this superfamily and is unique in its inhibitory specificity for calpains (10). It has been suggested that an imbalance between matrix metalloproteinases and tissue inhibitors of metalloproteinases could be responsible for cartilage breakdown in osteoarthritis (39). Calpastatin having been shown to be less abundant than calpains in the synovial fluid of RA patients (13), a disadvantageous enzyme/inhibitor ratio in some RA patients could also be associated with a more destructive potential of the arthri- 
tis. Antibodies to calpastatin could be entirely or partly responsible for this imbalance by binding to and somehow inactivating the inhibitor following its synthesis, expression on the plasma membrane, and/or secretion in vivo. This would result in both an immune complex-mediated inflammation and an uncontrolled activity of calpains.

One interesting observation is the discrepancy between the immunoreactivity of biochemically purified placental Sa antigen and of rRA-1. Indeed, absorption of sera with rRA-1 did not completely deplete their anti-Sa reactivity (data not shown). A number of explanations are possible. First, rRA-1 may lack epitopes found on the corresponding in vivo antigen: some epitopes may be situated on the $\mathrm{NH}_{2}$-terminal region of the protein that is not encoded by the RA-1 clone, or rRA-1 may lack antigenic posttranscriptional modifications. Second, some renaturation of the antigen blotted on nitrocellulose sheets is possible during immunoblotting; rRA-1 may thus lack some conformational epitopes found on the corresponding in vivo antigen. Third, anti-rRA-1 and anti-Sa antibodies may represent linked, but not identical, autoantibody systems, similar to anti-Ro and anti-La or to anti-U1 RNP and anti-Sm (1). The existence of anti-rRA-1 antibodies within sera of some patients without antiSa antibodies supports this last hypothesis. Careful definition of the epitope (s) recognized by anti-Sa and anti-RA-1 antibodies will be required to answer these questions.

The identification of calpastatin as an autoantigen in almost half of RA sera provide new research avenues. Studies are required to demonstrate the capacity of these antibodies to block the inhibitory activity of calpastatin on calpains. Furthermore, the presence of calpastatin-containing immune complexes should be demonstrated at the site of tissue damage in erosive RA. Finally, clinical correlation between the presence of anticalpastatin antibody and erosive disease will need to be systematically verified in RA and other rheumatic diseases, especially if an eventual therapeutic window is opened by synthetic cysteine proteinase inhibitors.

\section{Acknowledgments}

We thank Dr. Donald B. Bloch (Harvard Medical School, Boston, MA) for providing the human placenta cDNA library and for his invaluable help setting up the library screening. We thank Drs. Claude Asselin, Richard Leduc, and Mario S. Nemirovsky for critical advice and valuable suggestions; Moustapha El-Amine for providing the 3 ' noncoding region of the calpastatin mRNA; Maryse Hamel for excellent technical assistance; and Marthe Bégin for secretarial help.

This work was supported by the Medical Research Council of Canada (grant 10387 to H. A. Ménard). N. Després and G. Boire are supported by a studentship and a clinical associateship from The Arthritis Society, respectively.

\section{References}

1. Tan, E. M. 1991. Autoantibodies in pathology and cell biology. Cell. $67: 841-842$.

2. Burman, K. D., and J. R. Baker, Jr. 1985. Immune mechanisms in Graves' disease. Endocr. Rev. 6:183-232.

3. Schonbeck, S., S. Chrestel, and R. Hohlfeld. 1990. Myasthenia gravis: prototype of the antireceptor autoimmune diseases. Int. Rev. Neurobiol. 32:175200.

4. Carson, D. A. 1993. Rheumatoid factor. In Textbook of Rheumatology. W. N. Kelley, E. D. Harris, S. Ruddy, and C. B. Sledge, editors. W. B. Saunders Co., Philadelphia. 155-163.

5. Simon, M., E. Girbal, M. Sebbag, V. Gomès-Daudrix, C. Vincent,
G. Salama, and G. Serre. 1993. The cytokeratin filament-aggregating protein filaggrin is the target of the so-called "antikeratin antibodies," autoantibodies specific for rheumatoid arthritis. J. Clin. Invest. 92:1387-1393.

6. Steiner, G., K. Hartmuth, K. Skriner, I. Maurer-Fogy, A. Sinski, E. Thalmann, W. Hassfeld, A. Barta, and J. S. Smolen. 1992. Purification and partial sequencing of the nuclear autoantigen RA33 shows that it is indistinguishable from the A2 protein of the heterogeneous nuclear ribonucleoprotein complex. $J$. Clin. Invest. 90:1061-1066.

7. Hoet, R. M., A. M. Boerbooms, M. Arends, D. J. Ruiter, and W. J. van Venrooij. 1991. Antiperinuclear factor, a marker autoantibody for theumatoid arthritis: colocalization of the perinuclear factor and profilaggrin. Ann. Rheum. Dis. 50:611-618.

8. Després, N., G. Boire, F. J. Lopez-Longo, and H. A. Ménard. 1994. The Sa system: a novel antigen-antibody system specific for theumatoid arthritis. $J$. Rheumatol. 21:1027-1033.

9. Saitta, M. R., and J. D. Keene. 1992. Molecular biology of nuclear autoantigens. Rheum. Dis. Clin. North Am. 18:283-310.

10. Croall, D. E., and G. N. DeMartino. 1991. Calcium-activated neutral protease (calpain) system: structure, function, and regulation. Physiol. Rev. 71:813-847.

11. Suzuki, K., T. C. Saido, and S. Hirai. 1992. Modulation of cellular signals by calpain. Ann. N Y Acad. Sci. 674:218-227.

12. Squier, M. K., A. C. Miller, A. M. Malkinson, and J. J. Cohen. 1994 Calpain activation in apoptosis. J. Cell. Physiol. 159:229-237.

13. Yamamoto, S., K. Shimizu, K., Shimizu, K. Suzuki, Y. Nakagawa, and T. Yamamuro. 1992. Calcium-dependent cystein proteinase (calpain) in human arthritic synovial joints. Arthritis Rheum. 35:1309-1317.

14. Amett, F. C., S. M. Edworthy, D. A. Bloch, D. J. McShane, J. F. Fries, N. S. Cooper, L. A. Healey, S. R. Kaplan, M. H. Liang, H. S. Luthra, et al. 1988 The American Rheumatism Association 1987 revised criteria for the classification of rheumatoid arthritis. Arthritis Rheum. 31:315-324.

15. Prchal, J. T., B. J. Morley, S. H. Yoon, T. L. Coetzer, J. Palek, J. G. Conboy, and Y. W. Kan. 1987. Isolation and characterization of cDNA clones for human erythrocyte $\beta$-spectrin. Proc. Natl. Acad. Sci. USA. 84:7468-7472.

16. Sambrook, J., E. F. Fritsch, and T. Maniatis. 1989. Preparing stocks of bacteriophage $\lambda$ from single plaques. In Molecular Cloning: a Laboratory Manual. 2nd ed. Cold Spring Harbor Laboratory, Cold Spring Harbor, NY. 2.64-2.68.

17. Sanger, F., S. Miklen, and A. R. Coulson. 1977. DNA sequencing with chain termination inhibitors. Proc. Natl. Acad. Sci. USA. 74:5463-5467.

18. Smith, D. B., and K. S. Johnson. 1988. Single-step purification of peptides expressed in Escherichia coli as fusions with glutathione S-transferase. Gene. 67:31-40.

19. Asada, K., Y. Ishino, M. Shimada, T. Shimojo, M. Endo, F. Kimizuka, I. Kato, M. Maki, M. Hatanaka, and T. Murachi. 1989. cDNA cloning of human calpastatin: sequence homology among human, pig, and rabbit calpastatins. $J$. Enzyme Inhibtion 3:49-56.

20. Sherman, M. Yu., and A. L. Goldberg. 1992. Involvement of the chaperonin dnaK in the rapid degradation of a mutant protein in Escherichia coli. EMBO (Eur. Mol. Biol. Organ.) J. 11:71-77.

21. Naparstek, Y., and P. H. Plotz. 1993. The role of autoantibodies in autoimmune diseases. Annu. Rev. Immunol. 11:79-104.

22. Jackson, J. 1991. Autoantibody-facilitated proteolytic cleavage: a new pathogenic mechanism in autoimmunity. Biochem. Soc. Trans. 19:176-180.

23. van de Wiel, B. A., K. M. Dolman, C. H. van der Meer-Gerritsen, C. E. Hack, A. E. G. Kr. von dem Borne, and R. Goldschmeding. 1992. Interference of Wegener's granulomatosis autoantibodies with neutrophil proteinase 3 activity. Clin. Exp. Immunol. 90:409-414.

24. Dalpé, G., G. Boire, and H. A. Ménard. 1993. The conformational determinants recognized by Wegener's C-ANCAs are situated at or near the catalytic domain of myeloblastin. Clin. Exp. Immunol. 93(Suppl.):21.

25. Emori, Y., H. Kawasaki, S. Imajoh, Y. Minami, and K. Suzuki. 1988. All four repeating domains of the endogenous inhibitor for calcium-dependent protease independently retain inhibitory activity. Expression of the cDNA fragments in Escherichia coli. J. Biol. Chem. 263:2364-2370.

26. Poole, R. A. 1993. Cartilage in health and disease. In Arthritis and Allied Conditions: A Textbook of Rheumatology. 12th ed. D. J. McCarty and W. J. Koopman, editors. Lea \& Febiger, Malvern, PA. 279-333.

27. Buttle, D. J. 1993. Lysosomal cysteine endopeptidases in the degradation of cartilage and bone. In Textbook of Immunopharmacology: Immunopharmacology of Joints and Connective Tissue. J. T. Dingle and M. E. Davies, editors. Academic Press, New York. 225-243.

28. Lenarcic, B., D. Gabrijelcic, B. Rozman, M. Drobnic-Kosorok, and V. Turk. 1988. Human cathepsin B and cysteine proteinase inhibitors (CIPs) in inflammatory and metabolic joint diseases. Biol. Chem. Hoppe-Seyler 369(Suppl.):257-261.

29. Suzuki, K., K. Shimizu, T. Hamamoto, Y. Nakagawa, T. Murachi, and T. Yamamuro. 1992. Characterization of proteoglycan degradation by calpain Biochem. J. 285:857-862. 
30. Huet, G., R. M. Flipo, C. Richet, C. Thiebaut, D. Demeyer, M. Balduyck, B. Duquesnoy, and P. Degand. 1992. Measurement of elastase and cysteine proteinases in synovial fluid of patients with rheumatoid arthritis, sero-negative spondylarthropathies, and osteoarthritis. Clin. Chem. 38:1694-1697.

31. Suzuki, K., K. Shimizu, T. Hamamoto, Y. Nakagawa, T. Hamakubo, and T. Yamamuro. 1990. Biochemical demonstration of calpains and calpastatin in osteoarthritic synovial fluid. Arthritis Rheum. 33:728-732.

32. Fukui, I., K. Tanaka, and T. Murachi. 1989. Extracellular appearance of calpain and calpastatin in the synovial fluid of the knee joint. Biochem. Biophys. Res. Commun. 162:559-566.

33. Gabrijelcic, D., A. Annan-Prah, B. Rodic, B. Rozman, V. Cotic, and V. Turk. 1990. Determination of cathepsins $B$ and $H$ in sera and synovial fluids of patients with different joint diseases. J. Clin. Chem. Clin. Biochem. 28:149-153.

34. Maciewicz, R. A., and D. J. Etherington. 1988. Enzyme immunoassay for cathepsin $B$ and $L$ in synovial fluids from patients with arthritis. Biochem. Soc. Trans. 16:812-813.
35. Huet, G., R. M. Flipo, C. Colin, A. Janin, B. Hemon, M. C. D'Hooghe, R. Lafyatis, B. Duquesnoy, and P. Degand. 1993. Stimulation of the secretion of latent cysteine proteinase activity by tumor necrosis factor $\alpha$ and interleukin-1. Arthritis Rheum. 36:772-780.

36. Esser, R. E., R. A. Angelo, M. D. Murphey, L. M. Watts, L. P. Thornburg, J. T. Palmer, J. W. Talhouk, and R. E. Smith. 1994. Cysteine proteinase inhibitors decrease articular cartilage and bone destruction in chronic inflammatory arthritis. Arthritis Rheum. 37:236-247.

37. McCachren, S. S. 1991. Expression of metalloproteinases and metalloproteinases inhibitor in human arthritic synovium. Arthritis Rheum. 34:1085-1093.

38. Opdenakker, G., and J. Van Damme. 1992. Cytokines and proteases in invasive processes: molecular similarities between inflammation and cancer. $\mathrm{Cy}$ tokine. 4:251-258.

39. Dean, D. D., J. Martel-Pelletier, J.-P. Pelletier, D. S. Howell, and J. F. Woessner, Jr. 1989. Evidence for metalloproteinase and metalloproteinase inhibitor imbalance in human osteoarthritic cartilage. J. Clin. Invest. 84:678-685. 